\title{
Detecting network states in white noise
}

\author{
Jaroslav Hlinka ${ }^{1 *}$, Michal Hadrava ${ }^{1,2}$ \\ From 24th Annual Computational Neuroscience Meeting: CNS*2015 \\ Prague, Czech Republic. 18-23 July 2015
}

Nonstationarity of neural dynamics is a ubiquitous property that is crucial to understanding many key phenomena of both healthy and diseased brain function, including circadian rhythms, dynamics of epileptic activity as well as cognitive processing. Detecting switching of brain states has recently become of growing interest in the human brain neuroimaging community. However, from the data analysis/modelling perspective the task is quite challenging, and competing approaches exist [1]. One widely adopted approach is the use of clustering methods in the temporal domain to detect temporally contiguous clusters of time points with a similar structure of some instantaneous property - e.g. neural activity or functional connectivity profile. While this approach may in principle help to explore the switching structure of brain dynamics, it comes with technical challenges related the presence of noise in both the dynamics and measurements. In particular, as we documented in a recent study [2], comparison of the results with an appropriate null hypothesis is necessary to avoid spurious detection of nonstationarity markers such as switching of neural network states.

We document this danger by applying an example analysis pipeline used in [3] to simulated EEG datasets. The simulated data are generated as realizations of temporally white noise process (either spatially uncorrelated or spatially correlated in a pattern corresponding to real EEG data). In each case, one hundred realizations of a 5 seconds long epoch of $\mathrm{N}=20$ 'electrodes' (each of 2500 time points corresponding to $2 \mathrm{~ms}$ sampling rate). A kmeans clustering algorithm with $\mathrm{k}=2$ to 10 is applied to cluster the instantaneous synchronization likelihood matrix estimates with parameters as in [3]. The key observation stable across all setting is that the applied typical network switching analysis pipeline leads to spurious discovery of a multitude of network states in the stationary process realizations, with dominant state duration timescales of several tens to hundreds milliseconds qualitatively similar to the original results reported in [3]. These results suggest that observations of network switching should be always cautiously interpreted and tested against appropriate null models.

\section{Acknowledgements}

We thank Martin Brunovský from Psychiatric Center Prague for providing sample EEG data. The research was supported by the Czech Science Foundation projects No. 13-23940S and No. 13-17187S.

\section{Authors' details}

'Institute of Computer Science, Czech Academy of Sciences, Prague, Czech Republic. ${ }^{2}$ Faculty of Electrical Engineering, Department of Cybernetics, Czech Technical University in Prague, Prague, Czech Republic.

\section{Published: 18 December 2015}

\section{References}

1. Hutchison RM, Womelsdorf T, Allen EA, Bandettini PAD, Calhoun V, Corbetta M, Duyn JH, Glover GH, Gonzalez-Castillo J, et al: Dynamic functional connectivity: Promise, issues, and interpretations. Neurolmage 2013, 80:360-378.

2. Hlinka J, Hadrava M: On the danger of detecting network states in white noise. Frontiers in Computational Neuroscience 2015, 9:11.

3. Betzel RF, Abell M, O'Donnell BF, Hetrick WP, Sporns O: Synchronization dynamics and evidence for a repertoire of network states in resting EEG. Frontiers in Computational Neuroscience 2012, 6(74):1-13, doi:10.3389/ fncom.2012.00074.

\section{doi:10.1186/1471-2202-16-S1-P92}

Cite this article as: Hlinka and Hadrava: Detecting network states in white noise. BMC Neuroscience 2015 16(Suppl 1):P92.

\footnotetext{
* Correspondence: hlinka@cs.cas.cz

'Institute of Computer Science, Czech Academy of Sciences, Prague, Czech Republic

Full list of author information is available at the end of the article
} 